\title{
Paper spray mass spectrometry for high- throughput quantification of nicotine and cotinine $\uparrow$
}

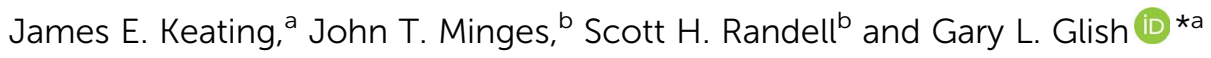

The rapid release of new tobacco products requires high-throughput quantitative methods to support tobacco research. Sample preparation for LC-MS and GC-MS is time consuming and limits throughput. Paper spray tandem mass spectrometry (PS-MS/MS) is proposed and validated as a simple and rapid method for quantification of nicotine and cotinine in complex matrices to support tobacco-related research. Air liquid interface (ALI) human tracheobronchial epithelial cell (HTBEC) cultures were exposed to tobacco smoke using a Vitrocell VC-10 smoking machine. Apical culture washes (phosphate buffered saline, PBS) and basolateral media were analyzed with the PS-MS/MS method. GC-MS/MS was used as a comparative quantitative technique. The PS-MS/MS approach allowed for direct spotting of samples on the paper substrate, whereas the GC-MS/MS method required additional sample preparation in the form of solvent-solvent extraction. Limits of quantitation (LOQs) were higher with the PS-MS/MS approach than GC-MS/MS, but still below the relevant concentrations found in HTBEC smoke exposure experiments as well as most clinical applications. PS-MS/MS is readily achieved on mass spectrometers that include atmospheric pressure inlets, and allows for convenient quantification from complex matrices that would otherwise require additional sample preparation and chromatographic separation.
\end{abstract}

Received 14th September 2017

Accepted 23rd November 2017

DOI: $10.1039 / c 7 a y 02204 b$

rsc.li/methods

\section{Introduction}

Mass spectrometry provides a highly sensitive and selective method of quantification for a wide variety of analyte molecules. In tobacco-related research, quantification of small molecules, especially those related to nicotine through structure or metabolism, is of particular interest, and has been well-established. ${ }^{1-3}$ Traditionally, the complexity of sample matrices relevant to tobacco research has required pre-treatment and/or chromatographic separation prior to mass spectrometric analysis., ${ }^{4,5}$ Gas chromatography-mass spectrometry (GC-MS) and liquid chromatography-mass spectrometry (LC-MS) are powerful techniques for separating analytes and interfering compounds, but are incompatible with high salt concentrations and matrix interferents inherent to the analysis of biological fluids. The use of chromatography also increases analysis time and adds complexity to the analytical system, requiring additional reagents and maintenance beyond what is required for mass spectrometry alone.

For rapid and convenient quantification it is preferable to bypass sample cleanup and chromatographic separations and introduce samples directly to the mass spectrometer. The recent

${ }^{a}$ Department of Chemistry, The University of North Carolina at Chapel Hill, Chapel Hill, NC 27599, USA. E-mail: glish@unc.edu

${ }^{b}$ Marsico Lung Inst. Cyst. Fibrosis Res. Ctr., The University of North Carolina at Chapel Hill, Chapel Hill, NC 27599, USA

$\dagger$ Electronic supplementary information (ESI) available. See DOI: 10.1039/c7ay02204b development of ambient ionization techniques has made this possible, and consequently these techniques have garnered a great deal of attention. ${ }^{6,7}$ Many of these techniques, including direct analysis in real time (DART) and desorption electrospray ionization (DESI), make it possible to directly analyze solids and surfaces. ${ }^{\mathbf{8}, 9}$ However, the nature of solid samples makes these ionization methods more suitable for qualitative or semiquantitative measurements than absolute quantification due to difficulty in preparing calibration standards. Paper spray ionization has been recognized as a powerful ambient ionization technique for direct analysis of solutions of complex matrices. ${ }^{10,11,17}$

The mechanism of paper spray is analogous to the widelyused electrospray ionization, with formation of a Taylor cone following application of solvent and high voltage to a sharptipped piece of paper. ${ }^{12}$ Droplet size and internal energy distributions of ions resulting from paper spray have been shown to be most similar to those generated by nanoelectrospray ionization. ${ }^{13}$ Sample $(1-50 \mu \mathrm{L})$ is spotted directly on a rigid piece of paper, allowed to dry, and analyzed following application of spray solvent (10-100 $\mu \mathrm{L})$ and high voltage (3.5$7 \mathrm{kV}$ ). Analyte is wicked towards the paper tip through multiple porous pathways via capillary action based on extraction compatibility with the spray solvent. Analyte wicking provides significant tolerance for interfering compounds and salts in the matrix and adds an extra degree of chemical specificity to the method. Single-use paper substrates mitigate carry-over often encountered in GC-MS and LC-MS approaches. This can 
improve limits of quantitation when working with analytes prone to carry-over on chromatographic systems. ${ }^{\mathbf{1 4 , 1 5}}$

Paper spray mass spectrometry (PS-MS) has previously been applied to the analysis of nicotine and related alkaloids in biofluids including blood, urine, and saliva. ${ }^{16}$ In vitro cytotoxicity assays are an important tool in tobacco research, enabling the testing of a large number of tobacco products over a range of experimental conditions that wouldn't be practical in vivo. ${ }^{17-19}$ Herein, quantification of nicotine and cotinine in matrices relevant to in vitro human tracheobronchial epithelial cell (HTBEC) tobacco exposure experiments was performed to further demonstrate the applicability of paper spray to tobacco research. These matrices, including the media for air-liquid interface (ALI) cultures (termed "ALI media") and phosphatebuffered saline (PBS), contain high salt concentrations (mM range) and the ALI media contains many cell growth supplements and additives, making them unsuitable for LC and GC, as well as direct analysis by most MS techniques. The compatibility of PS and complex matrices enabled the development of quantification methods using paper spray ionization and tandem mass spectrometry (MS/MS) for rapid and selective analysis of nicotine and cotinine. The developed PS-MS/MS methods were applied to samples from HTBEC cultures exposed to whole gas phase tobacco smoke in a highthroughput Vitrocell VC-10 smoking machine and compared to an extraction-based GC-MS/MS method.

\section{Experimental}

\section{Calibrators and samples}

Nicotine was purchased from MilliporeSigma (St. Louis, MO), and nicotine- $\mathrm{d}_{4}$, cotinine, and cotinine- $\mathrm{d}_{3}$ were purchased from Cerilliant (Round Rock, TX). Water (Optima grade), acetonitrile (Optima grade), and acetic acid (Optima grade) were purchased from Fisher Scientific (Hampton, NH). PBS and ALI media were prepared (ALI media protocol is given in $\mathrm{ESI} \dagger$ ) and used in preexposure HTBEC smoke exposure experiments as an apical lavage and basolateral cell media, respectively, to provide true matrix blanks for method development. Calibrators for nicotine, cotinine, and isotopically labeled standards were prepared in acetonitrile. To $60 \mu \mathrm{L}$ of pre-exposure PBS or ALI sample, $10 \mu \mathrm{L}$ of each standard was added and vortexed (30 s) for final nicotine and cotinine concentrations ranging from 10 to $1500 \mathrm{ng} \mathrm{mL} \mathrm{m}^{-1}$ and isotopically labeled standard concentrations of $1000 \mathrm{ng} \mathrm{mL} \mathrm{m}^{-1}$. Nicotine and cotinine in pre-exposure PBS and ALI were used directly in PS-MS/MS. Nicotine calibrators for GC-MS/MS were prepared by adjusting the $\mathrm{pH}$ to 13 by addition of 2.5 M NaOH followed by liquid-liquid extraction of PBS and ALI samples with $1 \mathrm{~mL}$ of $30: 30: 40$ methyl tert-butyl ether: dichloromethane : ethyl acetate, sample drying, and reconstitution in $80 \mu \mathrm{L}$ of acetonitrile. ${ }^{20}$

\section{Smoke exposures}

Briefly, ALI HTBEC $6.5 \mathrm{~mm}$ Transwell (Costar, 3470) cell cultures were created as previously described. ${ }^{21}$ Exposure to $3 \mathrm{R} 4 \mathrm{~F}$ reference cigarette smoke was performed in a Vitrocell
VC10 exposure system under ISO 3402 standard conditions (https://www.iso.org/standard/28324.html). Tobacco smoke was diluted with humidified air to final smoke percentages of $58.3 \%, 35.9 \%, 21.9 \%, 15.7 \%, 12.3 \%$, and $0 \%$ (air only). Cells from four non-smoking lung donors, procured under UNC Biomedical IRB-approved protocol \# 12-2293, were exposed to each smoke dilution in quadruplicate. Apical culture washings with PBS and conditioned ALI basolateral media were collected following tobacco smoke exposure. Samples were prepared for analysis by adding nicotine- $\mathrm{d}_{4}$ and cotinine- $\mathrm{d}_{3}$ to final concentrations of $1000 \mathrm{ng} \mathrm{mL} \mathrm{mL}^{-1}$. The solvent-solvent extraction method described above was used to further prepare exposure samples for GC-MS/MS analysis.

\section{Instrumentation}

PS-MS/MS experiments were performed with a Prosolia Velox 360 coupled to a Thermo Scientific LTQ-FT mass spectrometer. The mass spectrometer was set to recognize the Velox 360 as a nano-electrospray ionization source. GC-MS/MS experiments were performed on a Bruker EVOQ gas chromatograph-triple quadrupole mass spectrometer. A Vitrocell VC-10 smoking machine was used for in vitro HTBEC smoke exposure experiments.

\section{Instrument parameters}

Paper spray was performed by manually pipetting $10 \mu \mathrm{L}$ of sample onto the Velox sample cartridge. A solvent pump on the Velox 360 dispenses $100 \mu \mathrm{L}$ of $90: 10: 0.1 \%$ acetonitrile : water : acetic acid spray solution and spray voltage $(7.5 \mathrm{kV}$ for PBS, $5.5 \mathrm{kV}$ for ALI) is applied. MS/MS analysis was performed for 2 minutes per cartridge by monitoring the following transitions: $\mathrm{m} / \mathrm{z} 163.2$ to $\mathrm{m} / \mathrm{z} 132.2$ for nicotine, $\mathrm{m} / \mathrm{z} 167.2$ to $\mathrm{m} / \mathrm{z}$ 136.2 for nicotine- $\mathrm{d}_{4}, \mathrm{~m} / \mathrm{z} 177.2$ to $\mathrm{m} / \mathrm{z} 98.2$ for cotinine, and $\mathrm{m} / \mathrm{z}$ 180.2 to $\mathrm{m} / \mathrm{z} 101.2$ for cotinine- $\mathrm{d}_{3}$. The inlet capillary of the Thermo LTQ-FT was held at $200{ }^{\circ} \mathrm{C}$, and the sheath, auxiliary, and sweep gases were turned off. Mass spectrometer settings were chosen based on standard nESI source parameters.

GC-MS/MS was performed with $1 \mu \mathrm{L}$ splitless injections with an injector temperature of $280{ }^{\circ} \mathrm{C}$, transfer line temperature of $250{ }^{\circ} \mathrm{C}$, and electron ionization source temperature of $200{ }^{\circ} \mathrm{C}$. Helium (Airgas, 99.999\% purity) was used as a carrier gas with a linear velocity of $30 \mathrm{~cm} \mathrm{~s}^{-1}$. The oven temperature was ramped from 70 to $260{ }^{\circ} \mathrm{C}$ for a total run-time of 14 minutes. MS/MS analysis was performed by monitoring the following transitions: $m / z 162.2$ to $m / z 84.1$ for nicotine and $m / z 166.2$ to $\mathrm{m} / \mathrm{z}$ 84.1 for nicotine- $\mathrm{d}_{4}$.

\section{Results and discussion}

\section{PS-MS/MS method development}

The duration of a PS-MS/MS analysis is defined by application of high voltage to the Velox sample cartridge for the formation of electrospray. At the end of an experiment the voltage is turned off and the cartridge is discarded. Signal stability of the nicotine- $\mathrm{d}_{4}$ product ion $(\mathrm{m} / \mathrm{z}$ 136.2) during PS-MS/MS analysis is shown in Fig. 1. Signal intensity reaches a maximum 




Fig. 1 Extracted ion chronogram of nicotine- $\mathrm{d}_{4}$ product ion $(\mathrm{m} / \mathrm{z}$ 136.2) showing signal intensity over 2 minute PS-MS/MS analysis.

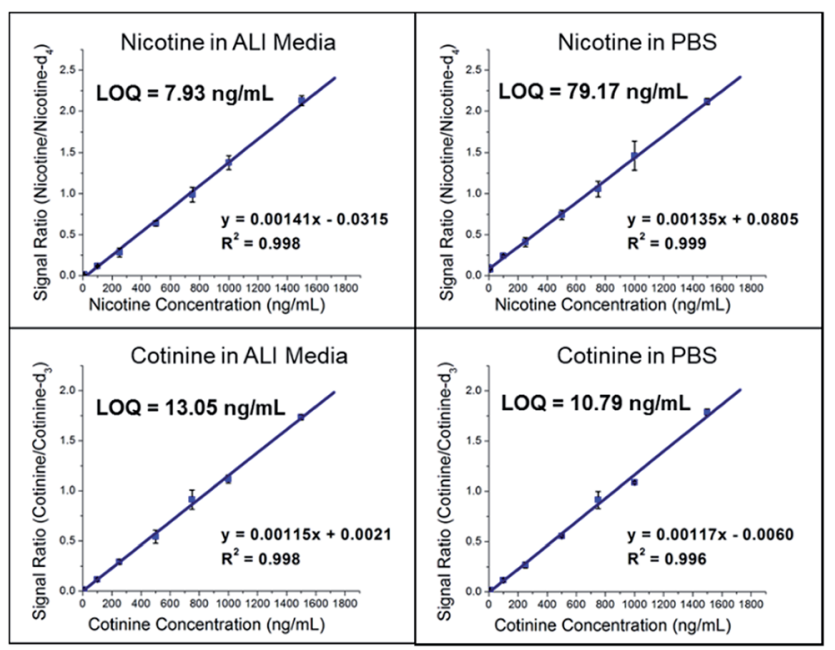

Fig. 2 Calibration curves (unweighted) of nicotine and cotinine in ALI and PBS from the pre-exposure HTBECs experiments using the Velox 360 and Thermo LTQ-FT. Bars represent standard deviation of triplicate measurements at each concentration. Limit of quantitation (LOQ) were calculated as $\frac{10 \sigma_{0}}{s}$, where $\sigma_{0}$ is the standard deviation of the blank (internal standard only) measurements and $s$ is the sensitivity of the method.

rapidly upon application of high voltage (HV) and decreases during analysis, eventually reaching zero intensity if $\mathrm{HV}$ remains on.
Application of $100 \mu \mathrm{L}$ of spray solvent generates signal for approximately 5 minutes before reaching zero intensity. This is attributed to loss and evaporation of the $90 / 10 / 0.1 \%$ acetonitrile : water: acetic acid spray solvent from the cartridge. Replenishing the spray solvent restores the signal intensity, and some custom-built paper spray sources include the ability to continuously add spray solvent for longer run times. ${ }^{22}$ The variation in signal intensity over time is corrected by using the ratio of analyte to isotopically labeled standard product ions for quantification, and for single-use cartridges a short run time is sufficient. Data analysis is performed by integrating the signal intensity over the 2 minute analysis for each analyte and isotopically labeled standard.

\section{PS-MS/MS and GC-MS/MS calibration}

Quantification of nicotine and cotinine in pre-exposure PBS and ALI was completed in triplicate using the PS-MS/MS methods developed and outlined above. Calibration (pre-exposure) matrices were designed to match the exposure sample matrices by collecting apical washes (PBS) and basal media (ALI) from HTBEC cells that had not been exposed to tobacco smoke. Matrix matching ensures consistency between calibrators and exposure samples, as materials from the cells are likely to be present after contact between the cells and solution. Calibrators for each analyte/matrix combination exhibited linearity within the entire calibration region found to be relevant to the HTBEC smoke exposure experiments. Plots for each are shown in Fig. 2. Limits of quantitation (LOQs) are calculated as $\frac{10 \sigma_{0}}{s}$, where $\sigma_{0}$ is the standard deviation of the blank (internal standard only) measurements and $s$ is the sensitivity of the method. Quantification of nicotine using the GC-MS/MS method was completed to provide results from a standard method to compare PS-MS/MS performance. LOQ's from the two methods are as follows: $79.17 \mathrm{ng} \mathrm{mL}^{-1}$ (PS-MS/MS) vs. $24.35 \mathrm{ng} \mathrm{mL}^{-1}$ (GC-MS/MS) for nicotine in PBS and $7.39 \mathrm{ng} \mathrm{mL}^{-1}$ (PS-MS/MS) vs. $5.42 \mathrm{ng} \mathrm{mL}^{-1}$ (GC-MS/MS) for nicotine in ALI.

An indirect comparison of the PS-MS/MS method to a clinically applicable test to distinguish smokers from non-smokers using cotinine concentrations in urine reports a cutoff of $49.7 \mathrm{ng} \mathrm{mL}{ }^{-1}$, which is well above LOQ's for cotinine in PBS

Table 1 Quantification of nicotine in the PBS apical lavages from HTBEC tobacco smoke exposure experiments using the developed PS-MS/MS and GC-MS/MS methods. ALI cultures from four donors were exposed to each concentration of tobacco smoke in quadruplicate and averaged to provide nicotine concentration and RSD values for each method

\begin{tabular}{|c|c|c|c|c|}
\hline $\begin{array}{l}\text { Tobacco smoke } \\
\text { concentration }^{a}\end{array}$ & $\begin{array}{l}\text { Concentration } \\
\left(\mathrm{ng} \mathrm{mL}^{-1}\right)\end{array}$ & RSD (\%) & $\begin{array}{l}\text { Concentration } \\
\left(\mathrm{ng} \mathrm{mL} \mathrm{mL}^{-1}\right)\end{array}$ & RSD (\%) \\
\hline $58.3 \%$ & 999 & 5.9 & 1060 & 28 \\
\hline $35.9 \%$ & 487 & 11 & 426 & 13 \\
\hline $21.9 \%$ & 234 & 16 & 175 & 21 \\
\hline $0 \%$ & 26 & 85 & 49 & 18 \\
\hline
\end{tabular}

${ }^{a} \%$ of tobacco smoke after dilution with laboratory air. 
(10.79 $\left.\mathrm{ng} \mathrm{mL}{ }^{-1}\right)$ and ALI (13.05 $\left.\mathrm{ng} \mathrm{mL}^{-1}\right) .{ }^{23}$ Urine was selected for comparison with ALI and PBS due to its high salt concentration, which is believed to be the main contributor to higher LOQs for the PS-MS/MS method over the GC-MS/MS method. As the relevant nicotine concentrations for the tobacco smoke exposure experiments are well above the LOQ's of both methods we conclude that PS-MS/MS is a suitable quantitative technique for these analyses. Unless an application requires the lower LOQ provided by the GC-MS/MS calibration experiment then the dramatically reduced sample preparation and analysis time of the PS-MS/MS method is preferable.

\section{PS-MS/MS and GC-MS/MS HTBEC smoke exposure results}

Nicotine in PBS apical lavages from the HTBEC smoke exposure experiments were quantified using the PS-MS/MS and GC-MS/ MS methods. Average concentrations $(n=4)$ for each dilution of tobacco smoke for each method along with associated uncertainty values (RSDs) appear in Table 1.

High RSD values $(>20 \%)$ are related to inconsistency of smoke exposure, rather than the quantitative methods. This is supported by the low RSD's (2-13\%) observed during calibration experiments using process matrix blanks of PBS.

To verify that the PS-MS/MS and GC-MS/MS methods are equivalent, a Passing-Bablok regression was used. ${ }^{24}$ PassingBablok tests for statistically significant differences in the slope and intercept from 1 and 0 , respectively, of a linear regression between results from two analytical methods. The linear relationship between the calibration results from each apical lavage sample $(n=24)$ from GC-MS/MS and PS-MS/MS $(x$ and $y$ variables, respectively) was confirmed using a cumulative sum test $(p=0.249)$.

Results from the Passing-Bablok model calculations are as follows: slope $=1.125(0.998$ to $1.292,95 \% \mathrm{CI})$ and intercept $=$ $8.4(-22.1$ to $33.5,95 \% \mathrm{CI})$. The bounds of the slope and intercept at the $95 \%$ confidence include the ideal values of 1 and 0 , suggesting that the results from the PS-MS/MS and GCMS/MS nicotine quantification from PBS apical lavages are statistically equivalent in the concentration range relevant to HTBEC smoke exposure.

\section{Conclusions}

Coupling high-throughput quantification with PS-MS/MS to high-throughput tobacco smoke exposure experiments with the Vitrocell VC-10 greatly increases the number of experiments that can be completed in a set period of time. Reducing the time required for sample preparation as well as analysis time of the method itself can permit screening of additional parameters for in vitro as well as in vivo studies (i.e. greater diversity of tobaccoproduct brands, concentration of tobacco smoke, etc.) to allow tobacco research to keep pace with the growing number of products. New and emerging tobacco products, especially electronic cigarettes (e-cigarettes) are of particular interest, in part because of the rapid release of new products as well as the relative lack of toxicity data. ${ }^{25-28}$
The validation of PS-MS/MS as a quantitative technique for analysis of nicotine and cotinine in complex matrices is a step towards a comprehensive toxicity screening platform with greater sampling capacity than what is available with current chromatography-based mass spectrometry methods. The simplicity of coupling PS to mass spectrometers with atmospheric pressure interfaces, which includes the vast majority of LC-MS instruments, makes it an attractive option for increasing sample throughput in tobacco-related research.

\section{Conflicts of interest}

There are no conflicts to declare.

\section{Acknowledgements}

This research was supported by the National Heart, Lung, and Blood Institute at the National Institute of Health (Grant \#5P50-HL-120100-01). We thank Prosolia Inc. for providing the Velox 360 PaperSpray source, Bruker Daltonics for providing the EVOQ 456 GC-MS platform, and Dr Russ Grant for helpful discussions.

\section{Notes and references}

1 B. H. Jung, B. C. Chung, S. J. Chung, M.-H. Lee and C. K. Shim, J. Pharm. Biomed. Anal., 1999, 20, 195-202.

2 J. T. Bernert, W. E. Turner, J. L. Pirkle, et al., Clin. Chem., 1997, 43, 2281-2291.

3 T. P. Moyer, J. R. Charlson, R. J. Enger, L. C. Dale, J. O. Ebbert, D. R. Schroeder and R. D. Hurt, Clin. Chem., 2002, 48, 1460-1471.

4 B. A. Tomkins, G. J. Van Berkel, R. A. Jenkins and R. W. Counts, J. Anal. Toxicol., 2006, 30, 178-186.

5 R. Dams, M. A. Huestis, W. E. Lambert and C. M. Murphy, J. Am. Soc. Mass Spectrom., 2003, 14, 1290-1294.

6 R. G. Cooks, Z. Ouyang, Z. Takats and J. M. Wiseman, Science, 2006, 311, 1566-1570.

7 M. Z. Huang, C. H. Yuan, S.-C. Cheng, Y.-T. Cho and J. Shiea, Annu. Rev. Anal. Chem., 2010, 3, 43-65.

8 R. B. Cody, J. A. Laramée and H. D. Durst, Anal. Chem., 2005, 77, 2297-2302.

9 Z. Takats, J. M. Wiseman, B. Gologan and R. G. Cooks, Science, 2004, 306, 471-473.

10 J. Liu, H. Wang, N. E. Manicke, J. M. Lin, R. G. Cooks and Z. Ouyang, Anal. Chem., 2010, 82, 2463-2471.

11 H. Wang, N. E. Manicke, Q. Yang, L. Zheng, R. Shi, R. G. Cooks and Z. Ouyang, Anal. Chem., 2011, 83, 11971201.

12 J. B. Fenn, M. Mann, C. K. Meng, S. F. Wong and C. M. Whitehouse, Science, 1989, 246, 64-71.

13 H. Wang, J. Liu, R. G. Cooks and Z. Ouyang, Angew. Chem., Int. Ed., 2010, 122, 889-892.

14 P. T. Vallano, S. B. Shugarts, E. J. Woolf and B. K. Matuszewski, J. Pharm. Biomed. Anal., 2005, 36, 10731078. 
15 R. Bakhtiar and T. K. Majumdar, J. Pharmacol. Toxicol. Methods, 2007, 55, 262-278.

16 H. Wang, Y. Ren, M. N. McLuckey, N. E. Manicke, J. Park, L. Zheng, R. Shi, R. G. Cooks and Z. Ouyang, Anal. Chem., 2013, 85, 11540-11544.

17 W. Sun, R. Wu and J. A. Last, Toxicology, 1995, 100, 163-174. 18 E. Elmore, T. T. Luc, V. E. Steele, G. J. Kelloff and J. L. Redpath, Methods Cell Sci., 2000, 22, 17-24.

19 F. Cervellati, X. M. Muresan, C. Sticozzi, R. Gambari, G. Montagner, H. J. Forman, C. Torricelli, E. Maioli and G. Valacchi, Toxicol. In Vitro, 2014, 28, 999-1005.

20 L. Carlos, F. Gomes, F. Oliveira and L. L. Pucci, Rev. Cienc. Farm. Basica Apl., 2013, 34, 177-182.
21 M. Leslie Fulcher, S. Gabriel, K. A. Burns, J. R. Yankaskas and S. H. Randell, in Human Cell Culture Protocols, Humana Press, New Jersey, 2005, pp. 183-206.

22 S. L. Reeber, S. Gadi, S.-B. Huang and G. L. Glish, Anal. Methods, 2015, 7, 9808-9816.

23 M. J. Jarvis, H. Tunstall-Pedoe, C. Feyerabend, C. Vesey and Y. Saloojee, Am. J. Public Health, 1987, 77, 1435-1438.

24 H. Passing and W. Bablok, Clin. Chem. Lab. Med., 1983, 21, 709.

25 B. M. Kuehn, J. Am. Med. Assoc., 2009, 302, 937.

26 D. L. Palazzolo, Front. Public Health, 2013, 1, 56.

27 M. J. Schroeder and A. C. Hoffman, Tob. Control, 2014, 23(2), 30-35.

28 R. Z. Behar, et al., Nicotine Tob. Res., 2014, 17, 1-8. 